\title{
Amphiphilic Block Copolymers of Polyvinyl Alcohol and Polystyrene and Their Surface Properties
}

\author{
Hong LI, Yong Ming ZHAng, ${ }^{\dagger}$ Ming Zhao XUE, and Yan Gang LiU \\ College of Chemistry and Chemical Technology, Shanghai Jiao Tong University, \\ Shanghai 200240, People's Republic of China
}

(Received June 8, 2005; Accepted July 20, 2005; Published November 15, 2005)

\begin{abstract}
Well-defined amphiphilic block copolymers of poly(vinyl alcohol)- $b$-polystyrene (PVA- $b$-PS) were successfully synthesized by atom transfer radical polymerization (ATRP) of styrene using trichloromethyl $\left(-\mathrm{CCl}_{3}\right)$ end grouped polyvinyl acetate as macroinitiators which were prepared via telomerization of vinyl acetate with carbon tetrachloride $\left(\mathrm{CCl}_{4}\right)$ as a telogen, followed by complete hydrolysis of polyvinyl acetate block. The block copolymers were characterized by GPC, FT-IR and ${ }^{1} \mathrm{H}$ NMR. PVA- $b$-PS has amphiphilic properties, which was confirmed with the study on the surface activity in $N, N^{\prime}$-dimethylformamide and toluene. And the Pearlescent pigment which was modified by PVA-b-PS shows improved dispersing stability. [DOI 10.1295/polymj.37.841]

KEY WORDS Amphiphilic Block Copolymer / Polyvinyl Alcohol / Surface Properties / Surface Modification / Pearlescent Pigment /
\end{abstract}

Amphiphilic block copolymers, such as poly(ethylene oxide)- $b$-poly(butyl acrylate) and poly(ethylene oxide)- $b$-polystyrene, which consist of at least two parts different chemical natures have recently attracted much attention due to the important applications in coatings, adhesives, pharmaceutical and photograph technologies, etc. ${ }^{1-4}$ Owing to their unique molecular structure, parallels can be drawn between typical surfactant and amphiphilic copolymers having both hydrophilic and hydrophobic blocks. Compared with low molecular surfactant, amphiphilic block copolymers have great advantage since through molecular design their surface properties can be controlled by the wide variability of the chemical structure, the length, and the ratio of hydrophobic and hydrophilic segments. So these polymers can substitute low molecular weight surfactant or extend surfactant applications in many heterophase stabilization problems, such as in emulsion polymerization and stabilization of pigments. ${ }^{5}$ Amphiphilic diblock or graft copolymer of poly(vinyl alcohol)-polystyrene (PVA-PS), which is combination of PVA and PS, two of the most commercially common homo-polymers, will be valuable either on academic research or commercial application. ${ }^{6 a, 6 b, 6 c}$

Atom transfer radical polymerization (ATRP) is one of the most effective tools for synthesis of block copolymers. ${ }^{7}$ A variety of monomers such as styrene, (meth)acrylate and acrylonitrile have been polymerized by ATRP. Due to the strong carbon-halogen bond at the chain-end of the PVAc formed, the equilibrium constant $\left(K_{\text {eq }}=k_{\text {act }} / k_{\text {deact }}\right)$ is too small. So, ATRP of vinyl acetate (VAc) is difficult, although some systems have produced the controlled PVAc via ATRP. ${ }^{8}$ To prepare polyvinyl acetate (PVAc) block copolymers, an alternative method is mechanistic transformation between ATRP and conventional radical polymerization. ${ }^{9,10}$ In this paper, vinyl acetate telomer having trichloromethyl end group (PVAc$\mathrm{CCl}_{3}$ ) was used as initiator to initiate the ATRP of styrene to obtain the block copolymer PVAc- $b$-PS. Then, the block copolymer was hydrolyzed to form amphiphilic copolymer poly(vinyl alcohol)- $b$-polystyrene (PVA- $b$-PS). Comparisons of the surface property and possible shape of the amphiphilic block copolymers arraying at the surface of polar and nonpolar solvents were studied. And surface modification of NY pearlescent pigment by PVA- $b$-PS was investigated.

\section{EXPERIMENTAL}

\section{Materials}

Styrene (AR, Shanghai Reagents Plant) was vacuum distilled above $\mathrm{CaH}_{2}$ before polymerization. Vinyl acetate (VAc) (AR, Shanghai Reagents Plant) was dried and distilled before use. $\mathrm{CuCl}$ (AR, Shanghai Reagents Plant) were purified according to literature. ${ }^{11}$ 2,2'-bipyridine (bpy) (AR, Shanghai Reagents Plant) was recrystallized from hexane and vacuum dried. PVAc-CCl $\mathrm{Cl}_{3}$ telomer $\left(M_{\mathrm{n}}=3766, M_{\mathrm{w}} / M_{\mathrm{n}}=\right.$ 1.51) was synthesized by telomerization of VAc with carbon tetrachloride as described in literature. ${ }^{9,12}$ Pearlescent pigment (Ling Bo Pigment Plant) was used as received.

\section{Preparation of Block Copolymers}

To a dried flask with magnetic bar, the PVAc- $-\mathrm{CCl}_{3}$

${ }^{\dagger}$ To whom correspondence should be addressed (Tel: +81-21-5474-5602, FAX: +81-21-5474-5602, E-mail: ymzhang@sjtu.edu.cn). 


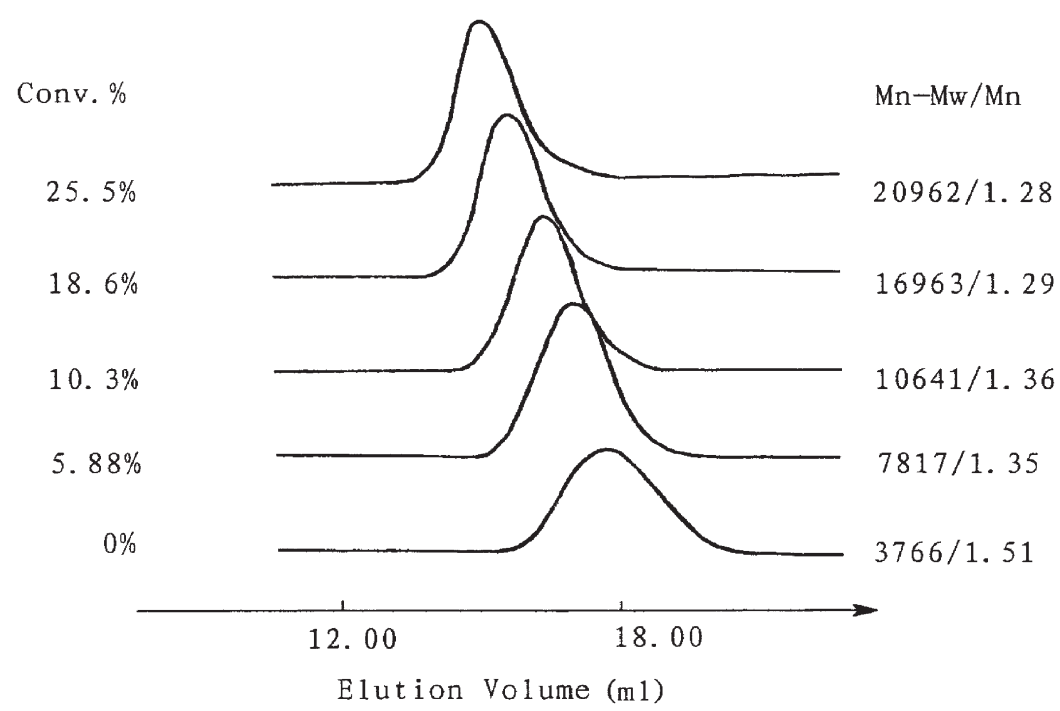

Figure 1. The GPC elution curves of PVAc- $b$-PS $\left[\mathrm{PVAc}-\mathrm{CCl}_{3}\right]:[\mathrm{CuCl}]:[\mathrm{bpy}]:[\mathrm{St}]_{0}=1: 1: 3: 660 ; 100{ }^{\circ} \mathrm{C}$; bulk.

$\left(1.33 \times 10^{-3} \mathrm{~mol}\right)$, bpy $\left(3.99 \times 10^{-3} \mathrm{~mol}\right)$ and $\mathrm{CuCl}$ $\left(1.33 \times 10^{-3} \mathrm{~mol}\right)$ were added respectively and degassed, followed by the introduction of the oxygenless styrene $\left(8.7 \times 10^{-1} \mathrm{~mol}\right)$. The mixture in the flask was degassed three times by "freeze-pump-thaw" cycles. The flask was put into an oil bath $\left(T=100^{\circ} \mathrm{C}\right)$. At timed intervals, samples were withdrawn using a degassed syringe, first for the measurement of the monomer conversion and then for the measurements of the molecular weight and the molecular weight distribution after passing a short $\mathrm{Al}_{2} \mathrm{O}_{3}$ column.

\section{Hydrolysis}

To a three-necked flask fitted with a mechanical stirrer, funnel and thermometer, the copolymer PVAc- $b$-PS $\left(0.51 \mathrm{~g}, M_{\mathrm{n}}=1.9 \times 10^{4} \mathrm{~g} / \mathrm{mol}\right.$, PVAc $/$ $\mathrm{PS}=1.18 / 0.72(\mathrm{w} / \mathrm{w}))$, menthol $(2 \mathrm{~mL})$, benzene $(8 \mathrm{~mL})$ and water $(0.2 \mathrm{~mL})$ were added. The block copolymer was dissolved at $60^{\circ} \mathrm{C}$. Then, sodium hydroxide $(6.25 \mathrm{mmol})$ was added. The reaction was stopped with a small amount of acetic acid. The product was precipitated in menthol, filtered and vacuum dried at $60^{\circ} \mathrm{C}$.

\section{Surface Modification of Pearlesent Pigment}

Interaction of surface of pearlecent pigment with PVA- $b$-PS was explored by immersing the pigment in PVA- $b$-PS toluene solution $\left(0.26 \times 10^{-3} \mathrm{M}\right)$ for $15 \mathrm{~min}$, filtering and drying in vacuum. After that, the pearlecent pigment was mixed with PS $(2 \%, \mathrm{w} \%)$.

\section{Characterization}

Monomer conversion was determined by gas chromatograph (GC). Molecular weight and molecular weight distribution were determined by gel permeation chromatography (GPC) using a waters 150C-

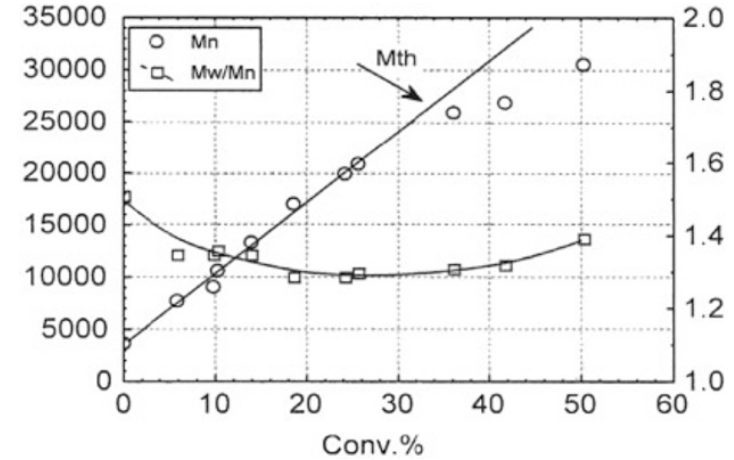

Figure 2. Plots of $M_{\mathrm{n}}$ and $M_{\mathrm{w}} / M_{\mathrm{n}} v s$. Monomer conversion in ATRP of St $\left[\mathrm{PVAc}-\mathrm{CCl}_{3}\right]:[\mathrm{CuCl}]:[\mathrm{bpy}]:[\mathrm{St}]_{0}=1: 1: 3: 660$; $100{ }^{\circ} \mathrm{C}$; bulk.

ALC/GPC with Waters Styrage ${ }^{\circledR}$ HT2, Styragel ${ }^{\circledR}$ HT4columns, and THF as an eluent. Polystyrene standards were used to calibrate the columns. FT-IR measurements and ${ }^{1} \mathrm{H}$ NMR characterization were performed on Nicolet Magna-IR-550 spectrometer and Gemini-300 ${ }^{1} \mathrm{H}$ NMR spectrometer, respectively. The surface tension of polymer solution was measured using the capillary rise method at $30^{\circ} \mathrm{C}$. SEM $(\mathrm{S}-250)$ was operated at $20 \mathrm{KV}$.

\section{RESULTS AND DISCUSSION}

\section{Synthesis of Poly(vinyl acetate)-b-polystyrene}

The $-\mathrm{CCl}_{3}$ end-grouped polyvinyl acetate, which was synthesized via telomerization of vinyl acetate with $\mathrm{CCl}_{4}$ as a telogen, was used as macroinitiator for atom transfer radical polymerization of styrene. GPC trace of the obtained polymers suggested the formation of the block copolymers without homopolymerization (Figure 1). 


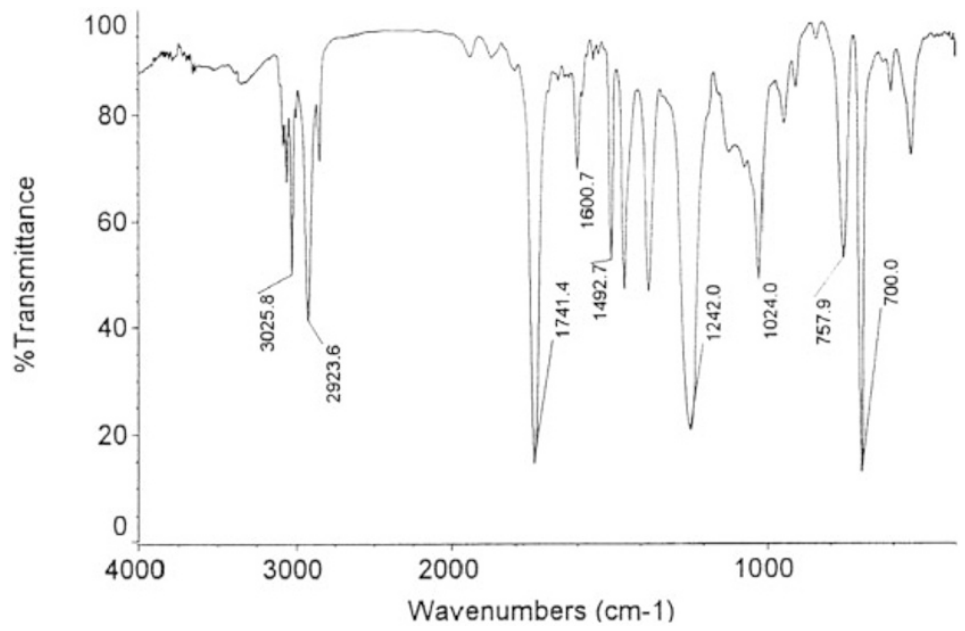

(a)

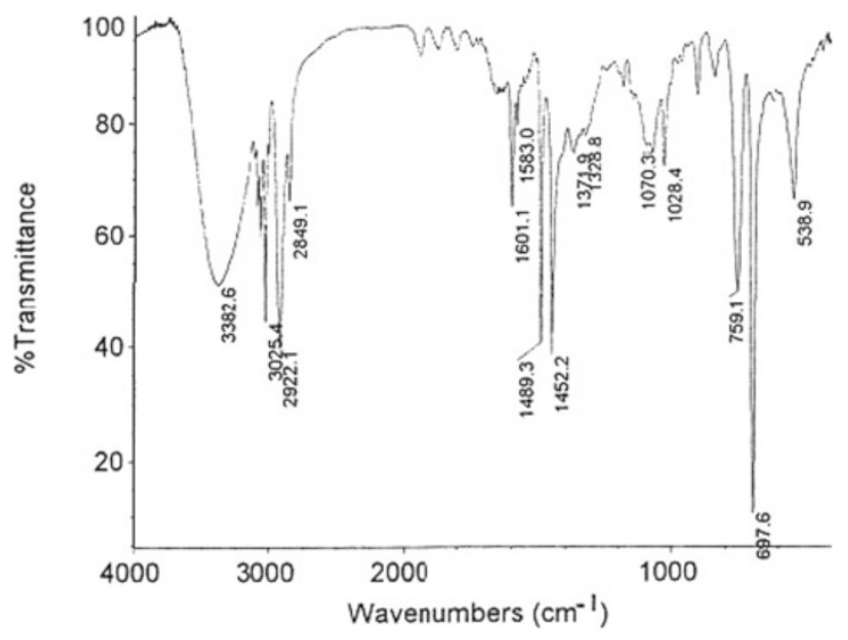

(b)

Figure 3. FT-IR spectra of PVAc- $b$-PS before (a) and after (b) hydrolysis.

Figure 2 shows that the theory molecular weight matches well with the molecular weight determined by GPC and the polydipersity is around 1.3 when the conversion of monomer is less than $30 \%$, which suggests the good control of polymerization. However, after that, the molecular weights deviated downward from the linear plot presumably due to the chain transfer reaction. ${ }^{13}$

The block copolymers were characterized by FT-IR and ${ }^{1} \mathrm{H}$ NMR. Figure 3 a shows the FT-IR spectrum of the block copolymer. The absorption band peaks at $700,758,1450,1492,1600$ and $3025 \mathrm{~cm}^{-1}$ attribute to the characteristic absorption of the phenyl ring. The absorbance of $\mathrm{C}=\mathrm{O}$ and $\mathrm{C}-\mathrm{O}$ was observed at 1741 and $1242 \mathrm{~cm}^{-1}$, respectively. Figure $4 \mathrm{a}$ shows the ${ }^{1} \mathrm{H}$ NMR spectrum of PVAc- $b$-PS. The aromatic protons resonances were observed at $6.6-7.4 \mathrm{ppm}$. Resonances for hydrogen nuclei at $\delta(\mathrm{ppm})$ of 1.9 $\left(\mathrm{CH}_{3} \mathrm{CO}-\right)$ and $5.1(-\mathrm{CH}-)$ for PVAc are shown in Figure 4a.

\section{Preparation of Amphiphilic Block Copolymer}

The hydrolysis of PVAc- $b$-PS was carried out in menthol and benzene with $\mathrm{NaOH}$ as catalyst. Compared with the FT-IR spectrum of PVAc- $b$-PS before hydrolysis (Figure 3a), the characteristic absorbance of $v_{\mathrm{O}-\mathrm{H}}$ from 2800 to $3600 \mathrm{~cm}^{-1}$ was observed in the spectrum of PVAc- $b$-PS after hydrolysis (Figure $3 b$ ) and the characteristic absorbance of $v_{\mathrm{C}=\mathrm{O}}$ in PVAc block at $1741 \mathrm{~cm}^{-1}$ was disappeared. As shown in ${ }^{1} \mathrm{H}$ NMR spectrum (Figure 4b), after hydrolysis the proton signal arising from the methyl of the acetyl group at $1.9 \mathrm{ppm}$ in the PVAc block had disappeared completely, and new peaks at 4.6-4.7 ppm, which are corresponding to hydroxyl groups, appeared. Thus, the hydrolysis of PVAc- $b$-PS is successful.

Surface Property of the Amphiphilic Block Copolymer in Nonpolar and Polar Solvent

PVA- $b$-PS molecule contains two different segments, the hydrophilic PVA segments and hydropho- 


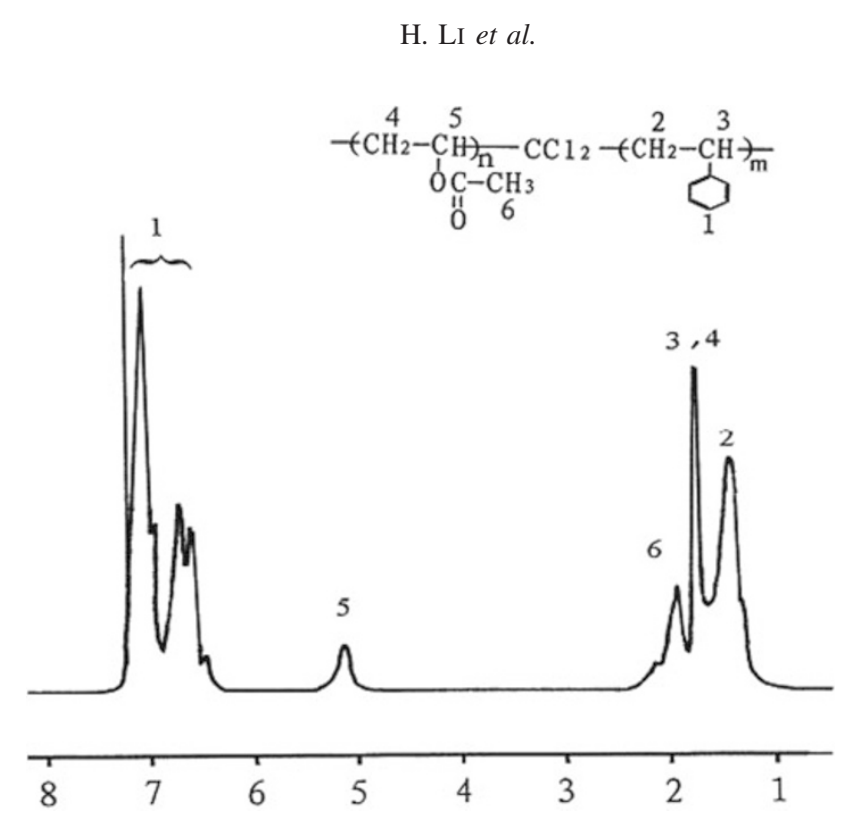

(a)

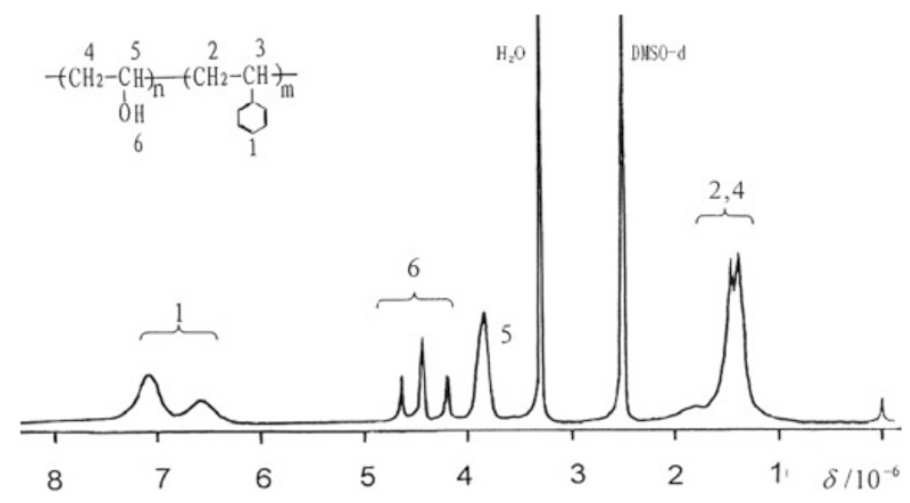

(b)

Figure 4. $\quad{ }^{1} \mathrm{H}$ NMR spectra of PVAc- $b$-PS before (a) and after (b) hydrolysis.

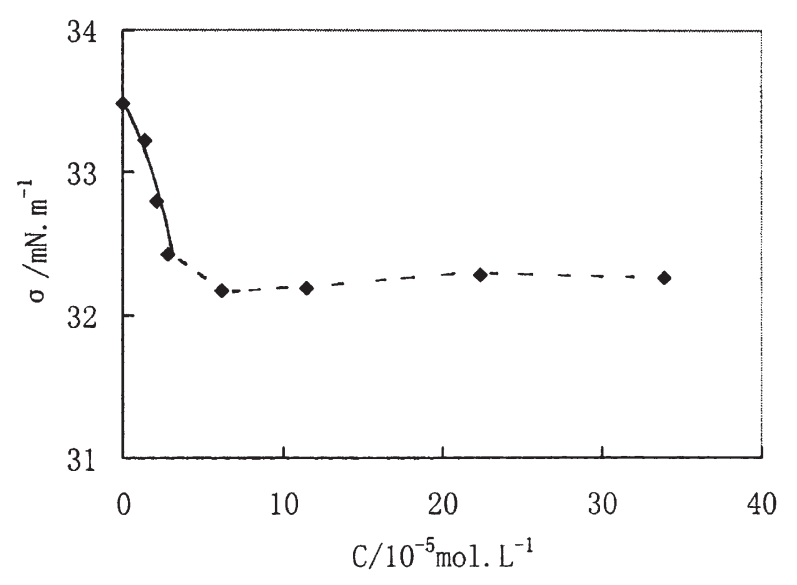

Figure 5. Surface tension of PVA- $b$-PS in DMF solution with different concentrations at $30^{\circ} \mathrm{C}$.

bic PS segments, which are the typical structure for the surfactant. Figure 5 shows the surface tension of PVA- $b$-PS $\left(M_{\mathrm{n}}=19832 \mathrm{~g} / \mathrm{mol}, M_{\mathrm{w}} / M_{\mathrm{n}}=1.28\right)$ in DMF solution with different concentrations at $30^{\circ} \mathrm{C}$. As shown in Figure 5, compared with the solvent

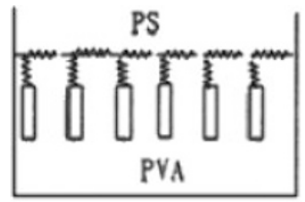

(a)

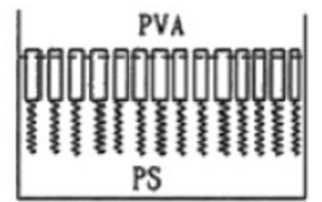

(b)
Scheme 1.

(DMF), the surface tension of the solution decreases with increasing the block copolymer concentration, which may due to that nonpolar PS block chain migrates to the surface of the $N, N^{\prime}$-dimethylformamide solution (Scheme 1a). Like low molecular surfactant, there is an inflection point above which the surface tension remains almost constant, which indicates the critical micelle concentration and occurs at $6.2 \times$ $10^{-5} \mathrm{~mol} \cdot \mathrm{L}^{-1}$. These phenomena may result from the aggregation behavior of the amphiphilic block copolymer PVA- $b$-PS in solution.

The data for $r-C$ curves in Figure 5 can be em- 
ployed in fitting an equation, i.e.

$$
r=r_{0}+a_{1} \ln C+a_{2}(\ln C)^{2}+a_{3}(\ln C)^{3} .
$$

Then $(d r / d \ln C)_{T}$ can be achieved from this equation and the absorption amount of block copolymer on the surface of solution $(\Gamma)$ at different copolymer concentrations can be calculated from Gibbs absorption equation: ${ }^{13}$

$$
\Gamma=-\frac{1}{R T}\left(\frac{d r}{d \ln C}\right)_{T}
$$

For dilute solution, eq 2 can be displaced with Langmuir equation:

$$
\Gamma=\Gamma_{m} \frac{b C}{1+b C}
$$

where, $b$ is a constant, $\Gamma_{m}$ is the saturate absorption amount of block copolymer on the surface of solution and can be calculated from the plot of $1 / C-1 / \Gamma$. According to eq 3 , the occupied area of one molecule on the surface $\left(A_{\mathrm{m}}\right)$ can be calculated:

$$
A_{\mathrm{m}}=\frac{1}{N_{\mathrm{A}} \Gamma_{m}}
$$

Where $N_{\mathrm{A}}$ is an Avogadro number. The values of $\Gamma_{m}$ and $A_{\mathrm{m}}$ are $8.47 \times 10^{-11} \mathrm{~mol} \cdot \mathrm{cm}^{-1}$ and $1.96 \mathrm{~nm}^{2}$, respectively. The sectional area of single PS chain is $0.03 \mathrm{~nm}^{2},{ }^{14}$ which is much less than $1.96 \mathrm{~nm}^{2}$. This indicated that the PS block of the copolymer may array folded at the surface of $N, N^{\prime}$-dimethylformamide solution (Scheme 1a). In DMF, the PS block, nonpolar segments, moved toward the surface caused by the repulsion from the polar solvent and then arranged at the surface of the solution.

PVA- $b$-PS consists of two different chain units (one is hydrophilic, the other is hydrophobic). So its interaction behavior with polar and nonpolar solvents would be different. Figure 6 shows the surface tension of PVA- $b$-PS in toluene solution with different concentrations at $30^{\circ} \mathrm{C}$. The surface tension of the solution decreases a little with increase of the amount of

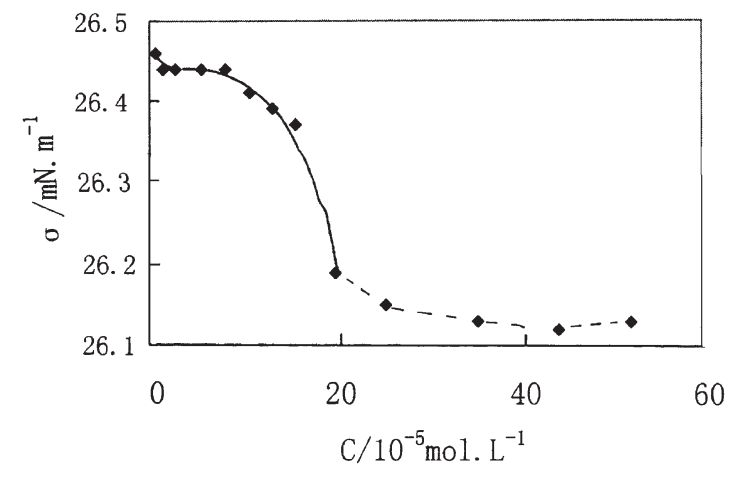

Figure 6. Surface tension of PVA- $b$-PS in toluene solution with different concentrations at $30^{\circ} \mathrm{C}$.

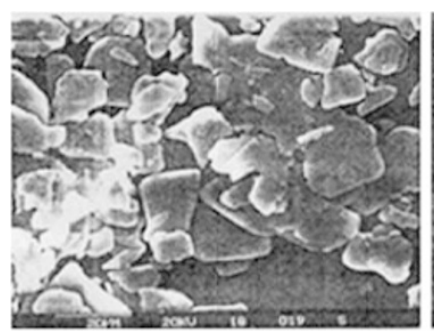

(a)

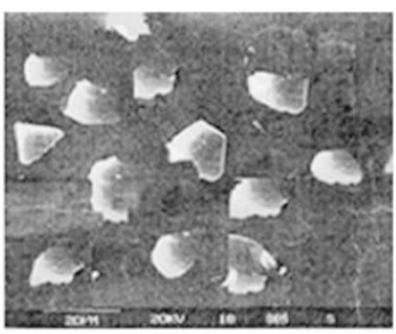

(b)
Figure 7. SEM photos of NY pearlescent pigments before (a) and after (b) surface modification by PVA- $b$-PS in PS.

PVA- $b$-PS. According to the Figure 6, the $\mathrm{cmc}$ of the solution is $2.2 \times 10^{-4} \mathrm{~mol} \cdot \mathrm{L}^{-1}$. The value $A_{\mathrm{m}}$ is $0.035 \mathrm{~nm}^{2}$, almost the same with the sectional area of single PS. This result indicated that the PS block of the copolymer may array vertically at the surface of toluene solution (Scheme 1b).

\section{Surface Modification of Pearlescent Pigment by PVA- $b$ - $P S$}

Cipolin pearlescent pigment, made by inorganic oxide such as $\mathrm{TiO}_{2}$ encapsulation on the surface of cipolin cores, is used in coating, plastic, rubber and cosmetic, and has high stability and optical property. However, due to dissociated hydroxyl groups on the surface of the pigment, it is difficulty to be wetted by nonpolar solvent and resin. And dispersing stability in such media does not satisfy the requirements. To improve the dispersing stability of pearlescent in polystyrene, PVA- $b$-PS was used as surface modifier.

Figure 7 shows SEM pictures of pearlescent pigment in polystyrene before and after surface modification. It is obvious that large aggregation in the system of pearlescent pigment before surface modification. By comparison of Figure $7 \mathrm{a}$, Figure $7 \mathrm{~b}$ shows that the pigments aggregates are dispersed. When pearlescent pigments adsorbed the PVA- $b$-PS, PVA block might anchor on the pigment, and PS block exposed on the outmost surface of the pigments. Since the surface energy of PS is much lower than that of $\mathrm{TiO}_{2}$, the particles after surface modification are easy to disperse. To further verify that dispersing stability of the modified pearlescent pigments by PVA- $b$-PS in PS was improved, SEM photo of the cross-section of PS film with NY pearlescent pigments was taken (Figure 8). From Figure 8a, the cross-section of PS film with NY pearlescent pigments before surface modification is rather rough, while that of the PS film with NY pearlescent pigments after surface modification by PVA- $b$-PS is smooth (Figure $8 \mathrm{~b}$ ). This indicates that after absorbing PVA- $b$-PS, NY pearlescent pigments have better consistency with PS resulting in improved dispersing stability in PS. 


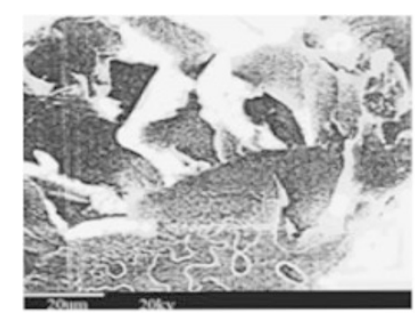

(a)

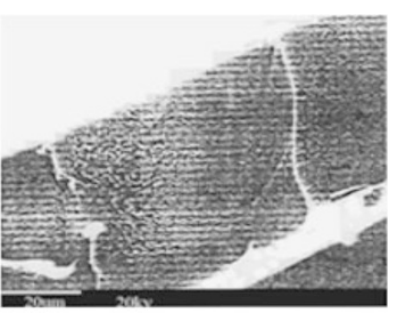

(b)
Figure 8. SEM photos of cross-section of PS film with NY pearlescent pigments before (a) and after (b) surface modification by PVA- $b$-PS.

\section{CONCLUSIONS}

Well-defined amphiphilic block copolymers poly(vinyl alcohol)- $b$-polystyrene were synthesized by ATRP and hydrolysis process. The surface properties of poly(vinyl alcohol)- $b$-polystyrene in $N, N^{\prime}$-dimethylformamide and toluene were examined and found to be quite different. The SEM measurement shows that dispersing stability of the modified pearlescent pigments by poly(vinyl alcohol)- $b$-polystyrene in polystyrene is increased.

\section{REFERENCES}

1. A. Ray, J. Am. Chem. Soc., 93, 7146 (1971).

2. J. R. Quintana, M. Villacampa, and I. A. Katime, Macromolecules, 26, 601 (1993).

3. D. Nguyen, C. E. Millians, and A. Eisenberg, Macromolecules, 27, 5060 (1994).

4. J. Fallbe, "Surfactants in Consumer Products," SpringerBerlag Heidelberg, New York, 1987.

5. S. Förster and M. Antonietti, Adv. Mater., 10, 195 (1998).

6. (a) Y. Tezuka and A. Araki, Langmuir, 10, 1865 (1994).

(b) Z. Pientka, H. Oike, and Y. Tezuka, Langmuir, 15, 3197 (1999).

(c) Y. Tezuka and A. Araki, Macromol. Chem. Phys., 194, 2827 (1993).

7. K. Matyjaszewski and J. H. Xia, Chem. Rev., 101, 2921 (2001).

8. M. Wakioka, K. Y. Baek, T. Ando, M. Kamigaito, and M. Sawamoto, Macromolecules, 35, 330 (2002).

9. M. Destarac, B. Pees, and B. Boutevin, Macromol. Chem. Phys., 201, 1189 (2000).

10. M. A. Semsarzadeh, A. Mirzaei, E. Vasheghani-Farahani, and M. N. Haghighi, Eur. Polym. J., 39, 2193 (2003).

11. N. R. Keller and D. H. Eycoff, Inorg. Synth., 2, 1 (1946).

12. Y. Tezuka, Macromol. Rapid Commun., 5, 559 (1984).

13. K. Matyjaszewski, Polym. Prepr., 37, 736 (1997).

14. M. J. Rosen, "Surfactants and Interfacial Phenomena," Wiley \& Sons, New York, N.Y., 1978. 